\title{
Blue-Emitting Small Silica Particles Incorporating ZnSe-Based Nanocrystals Prepared by Reverse Micelle Method
}

\author{
Masanori Ando, Chunliang Li, Ping Yang, and Norio Murase \\ Photonics Research Institute, National Institute of Advanced Industrial Science and Technology (AIST), Kansai Center, \\ 1-8-31 Midorigaoka, Ikeda 563-8577, Osaka, Japan \\ Correspondence should be addressed to Norio Murase, n-murase@aist.go.jp
}

Received 16 May 2007; Revised 17 December 2007; Accepted 24 December 2007

Recommended by Marek Osinski

ZnSe-based nanocrystals (ca. 4-5 $\mathrm{nm}$ in diameter) emitting in blue region (ca. $445 \mathrm{~nm}$ ) were incorporated in spherical small silica particles (20-40 $\mathrm{nm}$ in diameter) by a reverse micelle method. During the preparation, alkaline solution was used to deposit the hydrolyzed alkoxide on the surface of nanocrystals. It was crucially important for this solution to include $\mathrm{Zn}^{2+}$ ions and surfactant molecules (thioglycolic acid) to preserve the spectral properties of the final silica particles. This is because these substances in the solution prevent the surface of nanocrystals from deterioration by dissolution during processing. The resultant silica particles have an emission efficiency of $16 \%$ with maintaining the photoluminescent spectral width and peak wavelength of the initial colloidal solution.

Copyright () 2007 Masanori Ando et al. This is an open access article distributed under the Creative Commons Attribution License, which permits unrestricted use, distribution, and reproduction in any medium, provided the original work is properly cited.

\section{INTRODUCTION}

Development of bright and stable phosphors is increasingly required in many fields. The II-VI semiconductor nanocrystals (NCs) such as ZnSe and CdTe attract much interest as novel bright phosphors with tunable photoluminescence (PL) wavelength [1] for possible uses including biological labeling [2-6], display, and lighting devices [7-9]. For biological labeling, fluorophores are bound to biological molecules as fluorescent markers. It is possible to monitor the position and movement of virus and various materials by the PL of fluorophores. So far, organic fluorescent dyes were widely used for such biomarkers $[2,10]$. However, the emission wavelengths of these dyes are normally close to the excitation wavelengths, and therefore different excitation wavelengths are required for getting multiple colors. Moreover, deterioration and PL quenching of organic dyes occur in a short time of irradiation. For displays and lightings, currently commercialized phosphors are mostly rare earth ion-doped and transition metal ion-doped oxides [11]. These phosphors are highly stable; however, it is not easy to control the emission wavelength. In addition, they show a long decay time of PL (ca. 1 millisecond) because of a forbidden character of the transition. This slow decay causes the saturation of PL intensity when the excitation light intensity is increased.

The techniques to prepare highly luminescent and monodispersed semiconductor NCs have much advanced in this decade. They are organic solution method [12] and aqueous solution method [13]. Bright PL is obtained by capping the NCs with surfactants which deactivate the unpreferably PL-quenching surface defects. Aqueous solution method has several advantages over organic solution method: (1) highly luminescent NCs can be synthesized at lower temperature $\left(\sim 100^{\circ} \mathrm{C}\right)$ using a relatively safe and simple system; and that (2) the prepared water-dispersible NCs have higher stability of PL intensity in water and better compatibility with sol-gel fabrication.

We have reported the preparation of highly luminescent CdTe NCs [14] and ZnSe-based NCs [15-17] by the aqueous solution method. The PL wavelengths of these NCs depend on the band gap $\left(\mathrm{Eg}^{*}\right)$ which can be controlled by the composition and size of the NCs. The PL wavelength and efficiency of the NCs are almost independent of the excitation wavelength. The emission wavelength can be far from the excitation wavelength. Therefore, various PL colors are obtainable from various NCs using a single excitation 
wavelength. Compared with organic dyes, semiconductor NCs show much slower PL quenching or photobleaching on irradiation $[2,10]$. Furthermore, the II-VI semiconductor NCs have a direct transition band gap and fast PL decay in ca. 10 nanoseconds that is faster than those of rare earth ions and transition metal ions by ca. 5 orders of magnitude. This fast decay of PL leads to high brightness when the excitation light intensity is increased.

Recently, increasing attention has been directed to the semiconductor NCs incorporated in transparent matrices. The incorporation in silica matrices avoids the agglomeration of NCs and improves the long-term stability of PL. Previously, Mulvaney et al. reported the incorporation of NCs in $\mathrm{SiO}_{2}$ [18] and $\mathrm{ZrO}_{2}-\mathrm{SiO}_{2}$ [19] matrices by a solgel method. They used hydrophobic NCs (CdSe, CdSe/ZnS, and $\mathrm{CdSe} / \mathrm{CdS}$ ) prepared by organic solution method. However, water-dispersible NCs are more compatible with solgel method than such hydrophobic NCs. We have developed techniques to incorporate water-dispersible CdTe and $\mathrm{ZnSe}$ NCs in three forms of silica matrices (bulk $[16,20,21]$, small particles [22, 23], and thin films [24]) and in bulkform $\mathrm{Si}_{1-\mathrm{x}} \mathrm{Zr}_{\mathrm{x}} \mathrm{O}_{2}$ matrix [25], by using a sol-gel method. The prepared NC-incorporating phosphors showed bright PL of three primary colors. Among the three forms, small particles are expected to be used as fluorescent markers for biological labeling. Semiconductor NCs have already been reported to be applicable as fluorescent biomarkers [2-6]. There are several advantages in the incorporation of semiconductor NCs in small silica particles, such as protecting the NCs against oxidation and agglomeration, increasing the mechanical stability, and enabling a transfer into various organic and aqueous solvents. Furthermore, the surface of silica particles can be chemically modified to link bioconjugates.

Previously, several groups reported the preparation and PL efficiencies of silica particles incorporating emitting NCs, by chemical growth of silica shell around an NC (PL efficiency up to 18\%) [26]; by sol-gel formation of NCcontaining layer around a silica particle without containing NCs (PL efficiency up to 13\%) [27]; and by reverse micelle method (PL efficiency up to 20\%) [22, 23, 28-30]. The reverse micelle method is simpler than the chemical synthetic growth method and has wide controllability of NC concentration in the silica particles. When the NC concentration in a silica particle is high, both the increased emission brightness and decreased blinking of the silica particle can be expected. We have already reported green- and red-emitting silica particles incorporating CdTe NCs which were fabricated by the reverse micelle method $[22,23]$. The PL efficiencies reached $27 \%$ and $65 \%$, respectively, for green- and red-emitting silica particles. This PL efficiency $(65 \%)$ is the highest ever reported efficiency for the silica particles incorporating semiconductor NCs. In order to obtain PL of three primary colors for expanding the application range, blueemitting silica particles are required. However, bright blueemitting silica particles have not yet been reported to our knowledge. Zinc selenide NCs attract increasing interest because they can be synthesized in organic or aqueous solution and have tunable PL wavelength in blue region [15, 3133]. Recently, we synthesized highly luminescent ZnSe-based
NCs in blue region by an aqueous solution method $[16,17]$. The emission color of ZnSe NCs was shifted from blue-violet $(\sim 410 \mathrm{~nm})$ to pure blue $(\sim 440-485 \mathrm{~nm})$ by doping heavy elements such as $\mathrm{Cd}$ or Te, because, generally, the Eg* of II-VI semiconductor NCs becomes narrower when the constituent elements become heavier. Here we report the preparation of silica particle phosphor incorporating ZnSe-based NCs thus prepared by a reverse micelle method.

\section{MATERIALS AND METHODS}

\subsection{Chemicals}

All chemicals used were of analytical grade or of the highest purity available. Zinc perchlorate, cadmium perchlorate, and thioglycolic acid (TGA) were purchased from Sigma-Aldrich (Miss, USA). $\mathrm{Al}_{2} \mathrm{Se}_{3}$ lumps aquired from CERAC (Wiss, USA) were used to produce hydrogen selenide $\left(\mathrm{H}_{2} \mathrm{Se}\right)$ gas. Ammonia water and $\mathrm{NaOH}$ solution were purchased from Wako (Osaka, Japan). Deionized water $(18.3 \mathrm{M} \Omega$ ) was obtained from a Milli-Q water system (Millipore (Mass, USA)).

\subsection{Preparation of ZnSe-based NCs}

We have prepared core-shell ZnSe-based NCs by the previously reported aqueous solution method [15-17]. Briefly, the colloidal solutions of ZnSe-based NCs (core) were prepared using zinc perchlorate, cadmium perchlorate, and $\mathrm{H}_{2} \mathrm{Se}$ gas. TGA was used as the stabilizing surfactant that caps the NCs. The obtained weakly emitting Cd-doped ZnSe NCs $(\mathrm{Zn}: \mathrm{Cd}=95: 5$ (molar ratio upon synthesis)) was first dispersed in a solution containing cadmium perchlorate and TGA in the dark, and then irradiated with ultraviolet (UV) light $(365 \mathrm{~nm})$ in an aqueous solution containing zinc perchlorate and TGA [17]. The ZnS shell was formed on the Cddoped $\mathrm{ZnSe}$ core by the irradiation. Formation of this shell is effective for decreasing the number of surface defects and for increasing the robustness of NCs. As a result, strongly blueemitting Cd-doped ZnSe/ZnS NCs with a diameter of ca. 4$5 \mathrm{~nm}$ were prepared. The PL peak wavelength was $448 \mathrm{~nm}$ and PL efficiency was $49 \%$ in aqueous solution.

\subsection{Preparation of silica particles incorporating ZnSe-based NCs by reverse micelle method}

Silica particles incorporating ZnSe-based NCs were prepared by the procedure depicted in Scheme 1. Instead of using Solution 1 (diluted aqueous ammonia (6.25\%)) described in a previous report for preparing silica particles incorporating CdTe NCs [23], we used Solution 2 (Zn perchlorate, TGA, and $\mathrm{NaOH}$ solution $\left(\left[\mathrm{Zn}^{2+}\right]=0.261 \mathrm{~mol} / \mathrm{L}\right.$, molar ratio of $\left.\left.\left[\mathrm{Zn}^{2+}\right]:[\mathrm{TGA}]=1: 2.43, \mathrm{pH} \sim 11\right)\right)$ for nucleation of silica component to the surface of the particles in Step 1-1 and Step 3 . As described below, the Solution 2 was quite effective to retain the emission efficiency of the NCs.

Step 1-1. Aqueous solution of the NCs was mixed with alkaline solution of partially hydrolyzed tetraethyl orthosilicate (TEOS). Typically, $1 \mathrm{~mL}$ Cd-doped $\mathrm{ZnSe} / \mathrm{ZnS}$ colloidal solution $\left(\sim 3 \times 10^{-5} \mathrm{~mol}\right.$ particles/L), $1 \mathrm{~mL}$ water, $50 \mu \mathrm{L}$ alkaline 


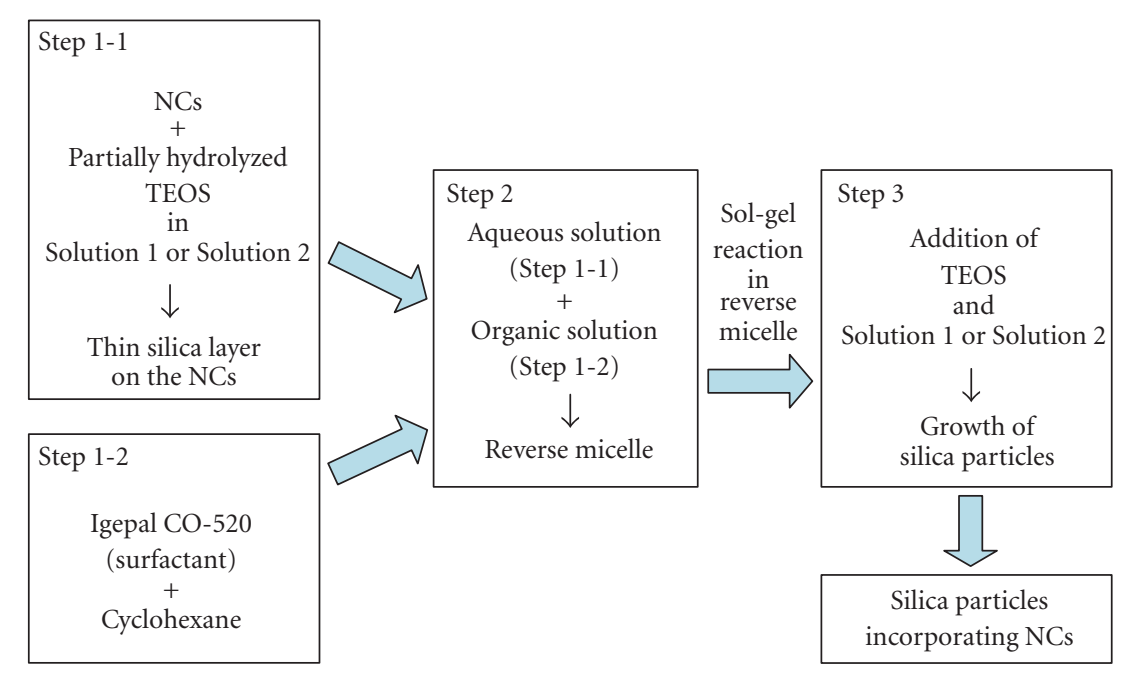

Solution 1: aqueous ammonia

Solution 2: $\mathrm{Zn}$ perchlorate, TGA and $\mathrm{NaOH}$ in water

Scheme 1: Preparation procedure of silica particles incorporating ZnSe-based NCs.

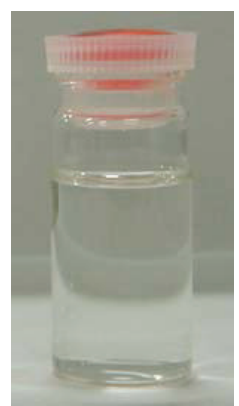

(a)

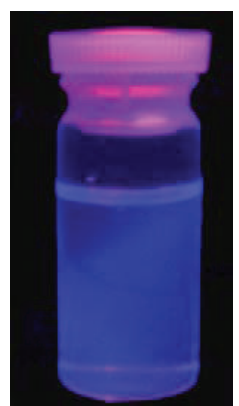

(b)

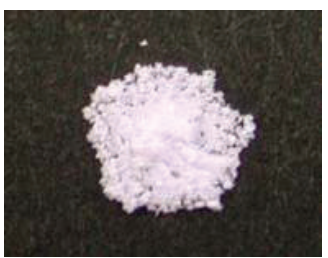

(c)

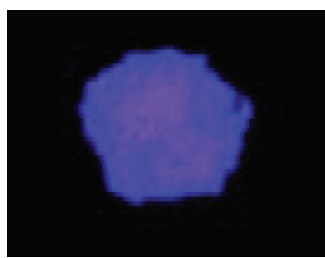

(d)

Figure 1: Appearance of silica particles incorporating ZnSe-based NCs prepared using Solution 2: (a) cyclohexane solution under visible light, (b) cyclohexane solution under UV light (wavelength: $365 \mathrm{~nm}$ ), (c) powder under visible light, and (d) powder under UV light (wavelength: $365 \mathrm{~nm}$ ).

solution (Solution 1 or 2 ), and $0.15 \mathrm{~mL}$ TEOS were mixed in a beaker. The $\mathrm{pH}$ of the mixed solution was 9-10. Then the solution was stirred for 2-3 hours to form thin silica layer on the surface of NCs [23].

Step 1-2. Nonionic surfactant Igepal CO-520 (polyoxyethylene(5)nonylphenyl ether) was dissolved in hydrophobic cyclohexane. Typically, $2.25 \mathrm{~g}$ Igepal CO-520 was dissolved in $12.5 \mathrm{~g}$ cyclohexane in a beaker.

Step 2. The aqueous solution prepared in Step 1-1 was injected dropwise to the cyclohexane solution prepared in Step 1-2. In this step, reverse micelles containing water droplets dispersing Cd-doped $\mathrm{ZnSe} / \mathrm{ZnS}$ NCs were formed.

Step 3. To grow outermost silica shell further, TEOS (typically $0.3 \mathrm{~mL}$ ) and alkaline aqueous solution (typically $100 \mu \mathrm{L}$ ) were added to the solution prepared in Step 2. Then the solution was stirred for 4 hours.

As a result, silica particles incorporating Cd-doped $\mathrm{ZnSe} / \mathrm{ZnS}$ NCs were obtained. These silica particles showed blue PL when irradiated with UV light. Transparent cyclohexane solution of silica particles incorporating NCs was obtained as supernatant by centrifuge of the solution at $4000 \mathrm{rpm}$.

For comparison, nonluminescent silica particles without containing NCs were prepared using Solution 1 in the similar manner. In Step 1-1, $1 \mathrm{~mL}$ diluted $\mathrm{NaOH}$ solution $(\mathrm{pH} \sim 11)$ was used instead of $1 \mathrm{~mL}$ Cd-doped $\mathrm{ZnSe} / \mathrm{ZnS}$ colloidal solution.

Size distribution of the obtained silica particles was measured by using a dynamic light scattering particle size analyzer (Nikkiso Microtrac Nanotrac 150). Transmission electron microscopy (TEM) of the silica particles was performed at an acceleration voltage of $300 \mathrm{kV}$ by using a Hitachi $\mathrm{H}$ 9000. For preparing a TEM specimen, the silica particles were extracted from the supernatant by precipitation with acetonitrile followed by drying in air at $\sim 40{ }^{\circ} \mathrm{C}$. The $\mathrm{PL}$ and absorption spectra of the silica particles were measured in 
cyclohexane by using conventional fluorescence spectrometer (Hitachi F-4500) and absorption spectrometer (Hitachi U-4000). The PL efficiencies of the solution samples (silica particles in cyclohexane and NCs in water) were estimated by comparison with standard solutions of quinine in aqueous $0.05 \mathrm{M} \mathrm{H}_{2} \mathrm{SO}_{4}$ solution (PL efficiency $=54.6 \%$ [34]).

\section{RESULTS AND DISCUSSION}

\subsection{Appearance of silica particles incorporating ZnSe-based NCs}

After centrifuge in the final step of preparation, the supernatant contained small silica particles and precipitate contained larger silica particles. Both supernatant and precipitate showed bright blue PL. The small silica particles were homogeneously dispersed in the supernatant (cyclohexane solution) at room temperature. The supernatant obtained by using Solution 2 showed brighter PL than that obtained by using Solution 1. Figures 1(a) and 1(b) show the appearance of the silica particles incorporating ZnSe-based NCs prepared using Solution 2 under visible light and under UV light, respectively. The cyclohexane solution was almost colorless and transparent under visible light (see Figure 1(a)) and emitted bright blue PL under UV light irradiation (see Figure 1(b)). On the other hand, the powder of small silica particles extracted from the above supernatant by using acetonitrile was white under visible light (see Figure 1(c)) and emitted blue PL under UV light (see Figure 1(d)).

\subsection{Sizes of silica particles incorporating ZnSe-based NCs}

Figure 2 shows the size (diameter) distribution of the small silica particles incorporating ZnSe-based NCs in the supernatant, measured by the dynamic light scattering method. The silica particles prepared using Solution 1 and those prepared using Solution 2 gave similar distribution curve of particle size, however, the latter particle size was a little smaller than the former one. Namely, the mean sizes of the silica particles prepared using Solution 1 and that using Solution 2 were ca. 35 and $31 \mathrm{~nm}$, respectively. These two kinds of silica particles had a spherical shape. Nonluminescent silica particles without containing NCs had similar size. A typical TEM photograph of the small silica particles incorporating $\mathrm{ZnSe}$ based NCs in the supernatant is shown in Figure 3. The shape of these small silica particles is spherical and the particle diameters are $20-40 \mathrm{~nm}$, which are in good agreement with those measured by the dynamic light scattering method (see Figure 2). On the other hand, the precipitate after centrifuge in the final step of preparation contained larger silica particles with diameters from a few hundreds nm to $1-2 \mu \mathrm{m}$. Such large silica particles also had a spherical shape. The results show that luminescent silica particles with various diameters were formed in the preparation process, and the size selection of silica particles was possible by centrifuge. It was also possible to select the size of silica particles by using syringe filters.

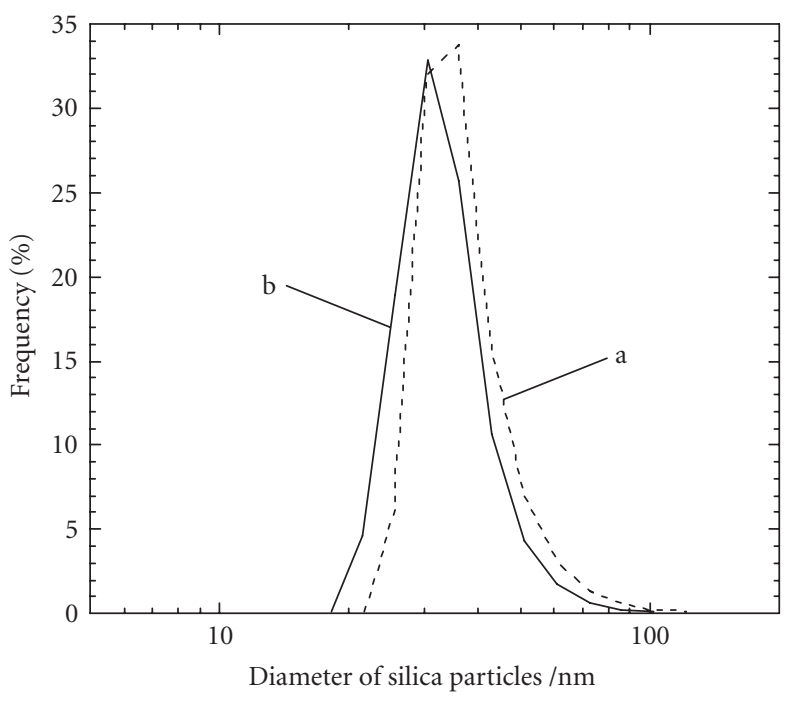

FIgURE 2: Size distribution of silica particles incorporating ZnSebased NCs measured by dynamic light scattering method: (a) silica particles prepared using Solution 1 and (b) silica particles prepared using Solution 2.

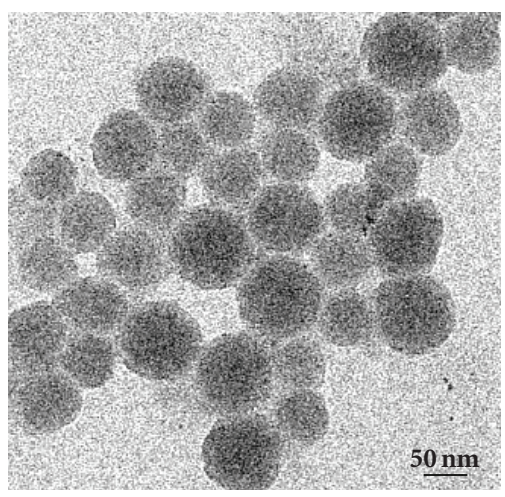

FIGURE 3: TEM photograph of silica particles incorporating ZnSebased NCs prepared using Solution 1.

\subsection{PL spectra, absorption spectra, and PL efficiencies of silica particles incorporating ZnSe-based NCs}

Figures 4 and 5 depict the PL spectra and absorption spectra of the small silica particles incorporating ZnSe-based NCs in the supernatant, respectively. The NC-incorporating silica particles prepared using Solution 2 showed an excitonic absorption of the NCs $[16,17]$ around $420-440 \mathrm{~nm}$. This absorption band was less clearly observed in the NCincorporating silica particles using Solution 1 and was not seen in the silica particles without incorporating NCs. This suggests that the deterioration of the NCs during incorporation in the silica particles was suppressed by using Solution 2. The PL peak of the NC-incorporating silica particles appeared in the absorption edge region. When the absorbance of the supernatant at the excitation wavelength $(350 \mathrm{~nm})$ was adjusted to the same value, the PL intensity of the silica particles prepared using Solution 2 was 2.3 times larger than that of the silica particles prepared using Solution 1 (see Figure 4). 


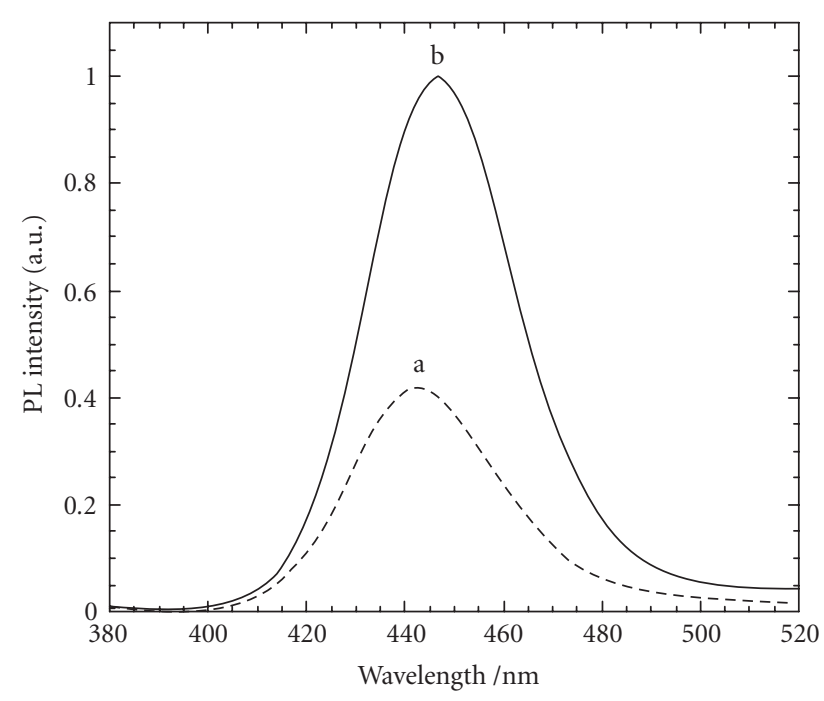

FIgURE 4: PL spectra of silica particles incorporating ZnSe-based NCs in solution: (a) silica particles prepared using Solution 1, (b) silica particles prepared using Solution 2. Excitation wavelength $=$ $350 \mathrm{~nm}$.

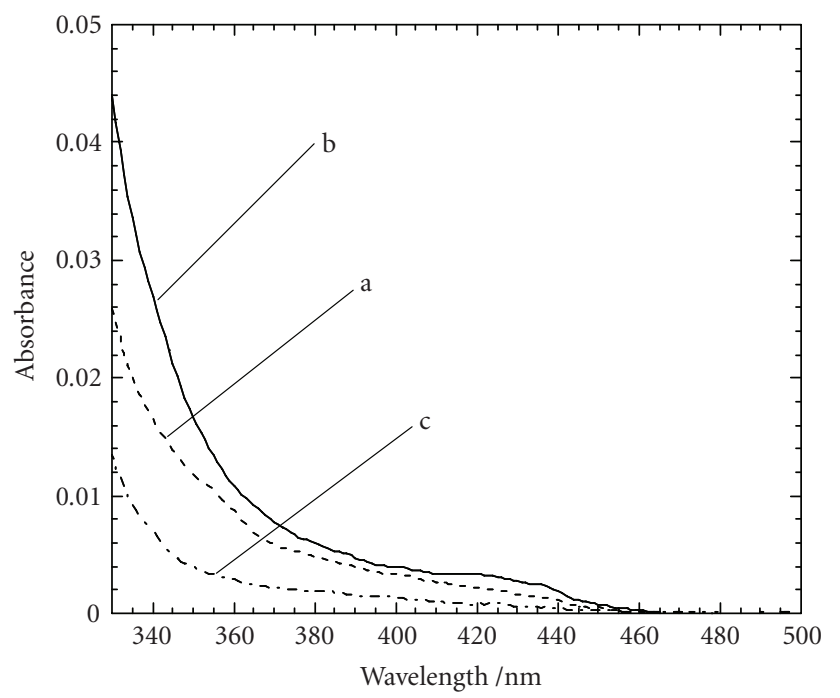

FIgURE 5: Absorption spectra of silica particles with and without ZnSe-based NCs in solution: (a) NC-incorporating silica particles prepared using Solution 1, (b) NC-incorporating silica particles prepared using Solution 2, and (c) silica particles without incorporating the NCs prepared using Solution 1.

As shown in Figure 4, these two PL spectra and that of the initial colloidal solution of NCs had almost the same shape. The widths of the PL spectra were also very close (Table 1). Compared with the initial colloidal solution of NCs, the silica particles showed small blue shift $(1-5 \mathrm{~nm})$ of the PL peak wavelength (Table 1). However, the blue shift in the silica particles prepared using Solution 2 was significantly smaller than that in the silica particles prepared using Solution 1. From the PL and absorption spectra, the PL efficiencies of the blue-emitting small silica particles using Solutions 1 and 2 were estimated to be 7 and $16 \%$, respectively (Table 1). As
TABLE 1: PL peak wavelength $\left(\lambda_{\mathrm{PL}}\right)$, spectral width (FWHM), and PL efficiency $(\eta)$ of silica particles incorporating the NCs together with the initial colloidal NCs.

\begin{tabular}{lccc}
\hline No. & (i) & (ii) & (iii) \\
\hline Sample & Silica particles & Silica particles & Initial NCs \\
Solution for & Solution 1 & Solution 2 & - \\
Steps 1-1 and 3 & & & - \\
$\lambda_{\mathrm{PL}} / \mathrm{nm}$ & 442.8 & 446.8 & 448.0 \\
$\mathrm{FWHM}^{\mathrm{a}} / \mathrm{nm}$ & 35.2 & 35.6 & 35.0 \\
$\eta(\%)$ & 7 & 16 & 49 \\
\hline
\end{tabular}

${ }^{\text {a }}$ Full width at half maximum.

exemplified by the preparation of silica phosphor dispersing CdTe NCs [20], the $\mathrm{Zn}^{2+}$ ions and TGA in Solution 2 prevent the surface of NCs from the deterioration during incorporation into silica particles. This leads to the almost unchanged PL wavelength and high PL efficiency. By contrast, when using the conventional aqueous ammonia (Solution 1), the observed larger blue shift of PL wavelength and lower PL efficiency were derived by deterioration of the NCs such as partial dissolution of the surface part into the surrounding media during incorporation into silica particles.

\section{CONCLUSION}

We have prepared bright blue-emitting silica particles incorporating core-shell Cd-doped $\mathrm{ZnSe} / \mathrm{ZnS} \mathrm{NCs}$ by a reverse micelle method. When using an alkaline solution containing zinc perchlorate and TGA during silica formation on the surface of NCs, the PL efficiency of the silica particles after preparation reached $16 \%$, which was 2.3 times larger than the PL efficiency of the silica particles prepared by using conventional aqueous ammonia. This is because the TGA-capped $\mathrm{ZnS}$ shell of the NCs is retained by the presence of zinc ions and TGA molecules during incorporation in silica particles. As we have already reported the preparation of green- and red-emitting silica particles, three primary emission colors are now obtainable from silica particles incorporating semiconductor NCs. These emitting silica particles are expected to be applicable as fluorescent biomarkers.

\section{ACKNOWLEDGMENT}

This study was supported in part by the Core Research for Evolutional Science and Technology (CREST) (research area: novel measuring and analytical technology contributions to the elucidation and application of life phenomena), sponsored by the Japan Science and Technology Agency (JST), Japan.

\section{REFERENCES}

[1] A. P. Alivisatos, "Perspectives on the physical chemistry of semiconductor nanocrystals," Journal of Physical Chemistry, vol. 100, no. 31, pp. 13226-13239, 1996.

[2] M. Bruchez Jr., M. Moronne, P. Gin, S. Weiss, and A. P. Alivisatos, "Semiconductor nanocrystals as fluorescent biological labels,” Science, vol. 281, no. 5385, pp. 2013-2016, 1998. 
[3] M. Han, X. Gao, J. Z. Su, and S. Nie, "Quantum-dot-tagged microbeads for multiplexed optical coding of biomolecules," Nature Biotechnology, vol. 19, no. 7, pp. 631-635, 2001.

[4] W. C. W. Chan, D. J. Maxwell, X. Gao, R. E. Bailey, M. Han, and S. Nie, "Luminescent quantum dots for multiplexed biological detection and imaging," Current Opinion in Biotechnology, vol. 13, no. 1, pp. 40-46, 2002.

[5] W. J. Parak, D. Gerion, T. Pellegrino, et al., "Biological applications of colloidal nanocrystals," Nanotechnology, vol. 14, no. 7, pp. R15-R27, 2003.

[6] X. Michalet, F. F. Pinaud, L. A. Bentolila, et al., "Quantum dots for live cells, in vivo imaging, and diagnostics," Science, vol. 307, no. 5709, pp. 538-544, 2005.

[7] N. P. Gaponik, D. V. Talapin, A. L. Rogach, and A. Eychmüller, "Electrochemical synthesis of CdTe nanocrystal/polypyrrole composites for optoelectronic applications," Journal of Materials Chemistry, vol. 10, no. 9, pp. 2163-2166, 2000.

[8] S. Coe, W.-K. Woo, M. Bawendi, and V. Bulović, "Electroluminescence from single monolayers of nanocrystals in molecular organic devices," Nature, vol. 420, no. 6917, pp. 800-803, 2002.

[9] Y. Mori, Y. Arao, K. Tsuchiya, and K. Shimamura, "Photoluminescence properties of $\mathrm{ZnS}$ nanoparticles prepared in clay suspension," in Proceedings of the 58th Divisional Meeting on Colloid and Interface Chemistry, p. 300, Utsunomiya, Japan, September 2005.

[10] D. Toomre and D. J. Manstein, "Lighting up the cell surface with evanescent wave microscopy," Trends in Cell Biology, vol. 11, no. 7, pp. 298-303, 2001.

[11] Phosphor Research Society, Ed., Handbook of Phosphors, Ohmsha, Tokyo, Japan, 1987.

[12] B. O. Dabbousi, J. Rodriguez-Viejo, F. V. Mikulec, et al., "(CdSe) ZnS core-shell quantum dots: synthesis and characterization of a size series of highly luminescent nanocrystallites," Journal of Physical Chemistry B, vol. 101, no. 46, pp. 94639475, 1997.

[13] A. L. Rogach, L. Katsikas, A. Kornowski, D. S. Su, A. Eychmüller, and H. Weller, "Synthesis and characterization of thiol-stabilized CdTe nanocrystals," Berichte der BunsenGesellschaft-Physical Chemistry, vol. 100, pp. 1772-1778, 1996.

[14] C. Li and N. Murase, "Surfactant-dependent photoluminescence of CdTe nanocrystals in aqueous solution," Chemistry Letters, vol. 34, no. 1, pp. 92-93, 2005.

[15] N. Murase and M. Gao, "Preparation and photoluminescence of water-dispersible ZnSe nanocrystals," Materials Letters, vol. 58, no. 30, pp. 3898-3902, 2004.

[16] C. Li, K. Nishikawa, M. Ando, H. Enomoto, and N. Murase, "Highly luminescent water-soluble ZnSe nanocrystals and their incorporation in a glass matrix," Colloids and Surfaces A, vol. 294, no. 1-3, pp. 33-39, 2007.

[17] C. Li, K. Nishikawa, M. Ando, H. Enomoto, and N. Murase, "Blue-emitting type-II semiconductor nanocrystals with high efficiency prepared by aqueous method," Chemistry Letters, vol. 36, no. 3, pp. 438-439, 2007.

[18] S. T. Selvan, C. Bullen, M. Ashokkumar, and P. Mulvaney, "Synthesis of tunable, highly luminescent QD-glasses through sol-gel processing," Advanced Materials, vol. 13, no. 12-13, pp. 985-988, 2001.

[19] C. Bullen, P. Mulvaney, C. Sada, M. Ferrari, A. Chiasera, and A. Martucci, "Incorporation of a highly luminescent semiconductor quantum dot in $\mathrm{ZrO}_{2}-\mathrm{SiO}_{2}$ hybrid sol-gel glass film," Journal of Materials Chemistry, vol. 14, no. 7, pp. 1112-1116, 2004.
[20] C. Li and N. Murase, "Synthesis of highly luminescent glasses incorporating CdTe nanocrystals through sol-gel processing," Langmuir, vol. 20, no. 1, pp. 1-4, 2004.

[21] C. Li, M. Ando, and N. Murase, "Preparation and characterization of glass embedding photoluminescent CdTe nanocrystals," Journal of Non-Crystalline Solids, vol. 342, no. 1-3, pp. 32-38, 2004.

[22] S. T. Selvan, C. Li, M. Ando, and N. Murase, "Formation of luminescent CdTe-silica nanoparticles through an inverse microemulsion technique," Chemistry Letters, vol. 33, no. 4, pp. 434-435, 2004.

[23] P. Yang, M. Ando, and N. Murase, "Encapsulation of emitting CdTe QDs within silica beads to retain initial photoluminescence efficiency," Journal of Colloid and Interface Science, vol. 316, no. 2, pp. 420-427, 2007.

[24] P. Yang, C. Li, and N. Murase, "Highly photoluminescent multilayer QD-glass films prepared by LbL self-assembly," Langmuir, vol. 21, no. 19, pp. 8913-8917, 2005.

[25] P. Yang and N. Murase, "Intensely emitting CdTe nanocrystals retained initial photoluminescence efficiency in sol-gel derived $\mathrm{Si}_{1-\mathrm{x}} \mathrm{Zr}_{\mathrm{x}} \mathrm{O}_{2}$ glass," Applied Physics A, vol. 89, no. 1, pp. 189193, 2007.

[26] D. Gerion, F. Pinaud, S. C. Williams, et al., "Synthesis and properties of biocompatible water-soluble silica-coated CdSe/ZnS semiconductor quantum dots," Journal of Physical Chemistry B, vol. 105, no. 37, pp. 8861-8871, 2001.

[27] Y. Chan, J. P. Zimmer, M. Stroh, J. S. Steckel, R. K. Jain, and M. G. Bawendi, "Incorporation of luminescent nanocrystals into monodisperse core-shell silica microspheres," Advanced Materials, vol. 16, no. 23-24, pp. 2092-2097, 2004.

[28] S. T. Selvan, T. T. Tan, and J. Y. Ying, "Robust, non-cytotoxic, silica-coated CdSe quantum dots with efficient photoluminescence," Advanced Materials, vol. 17, no. 13, pp. 1620-1625, 2005.

[29] Y. Yang and M. Gao, "Preparation of fluorescent $\mathrm{SiO}_{2}$ particles with single CdTe nanocrystal cores by the reverse microemulsion method," Advanced Materials, vol. 17, no. 19, pp. 23542357, 2005.

[30] M. Darbandi, R. Thomann, and T. Nann, "Single quantum dots in silica spheres by microemulsion synthesis," Chemistry of Materials, vol. 17, no. 23, pp. 5720-5725, 2005.

[31] X. Zhong, M. Han, Z. Dong, T. J. White, and W. Knoll, "Composition-tunable $\mathrm{Zn}_{x} \mathrm{Cd}_{1-x}$ Se nanocrystals with high luminescence and stability," Journal of the American Chemical Society, vol. 125, no. 28, pp. 8589-8594, 2003.

[32] R. Li, J. Lee, D. Kang, Z. Luo, M. Aindow, and F. Papadimitrakopoulos, "Band-edge photoluminescence recovery from zinc-blende CdSe nanocrystals synthesized at room temperature," Advanced Functional Materials, vol. 16, no. 3, pp. 345350, 2006.

[33] Q. Zhao and W. Xu, "One-step preparation of ZnSe nanorod aggregates," Chemistry Letters, vol. 35, no. 10, pp. 1186-1187, 2006.

[34] D. F. Eaton, "Reference materials for fluorescence measurement," Pure and Applied Chemistry, vol. 60, no. 7, pp. 11071114, 1988. 

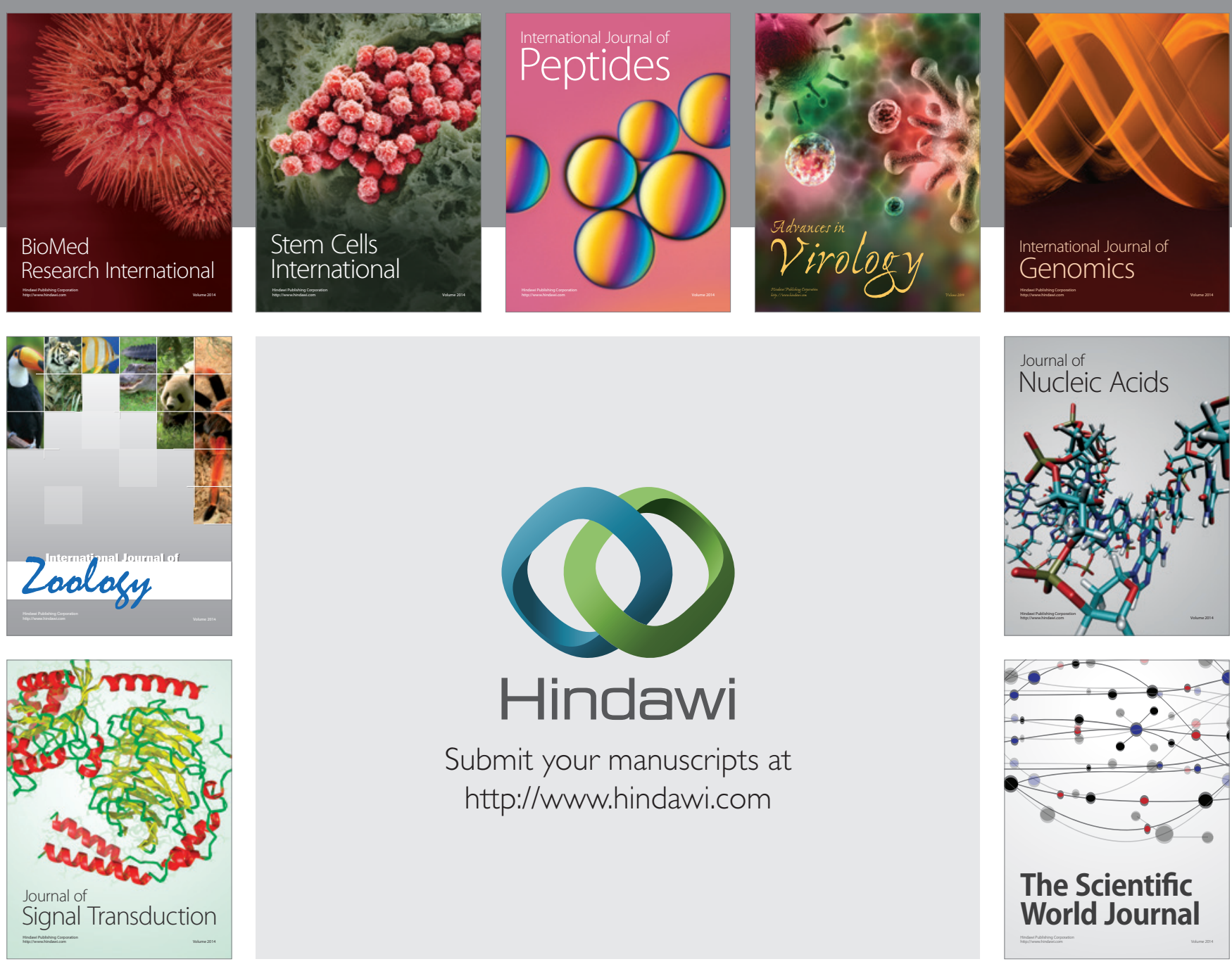

Submit your manuscripts at

http://www.hindawi.com
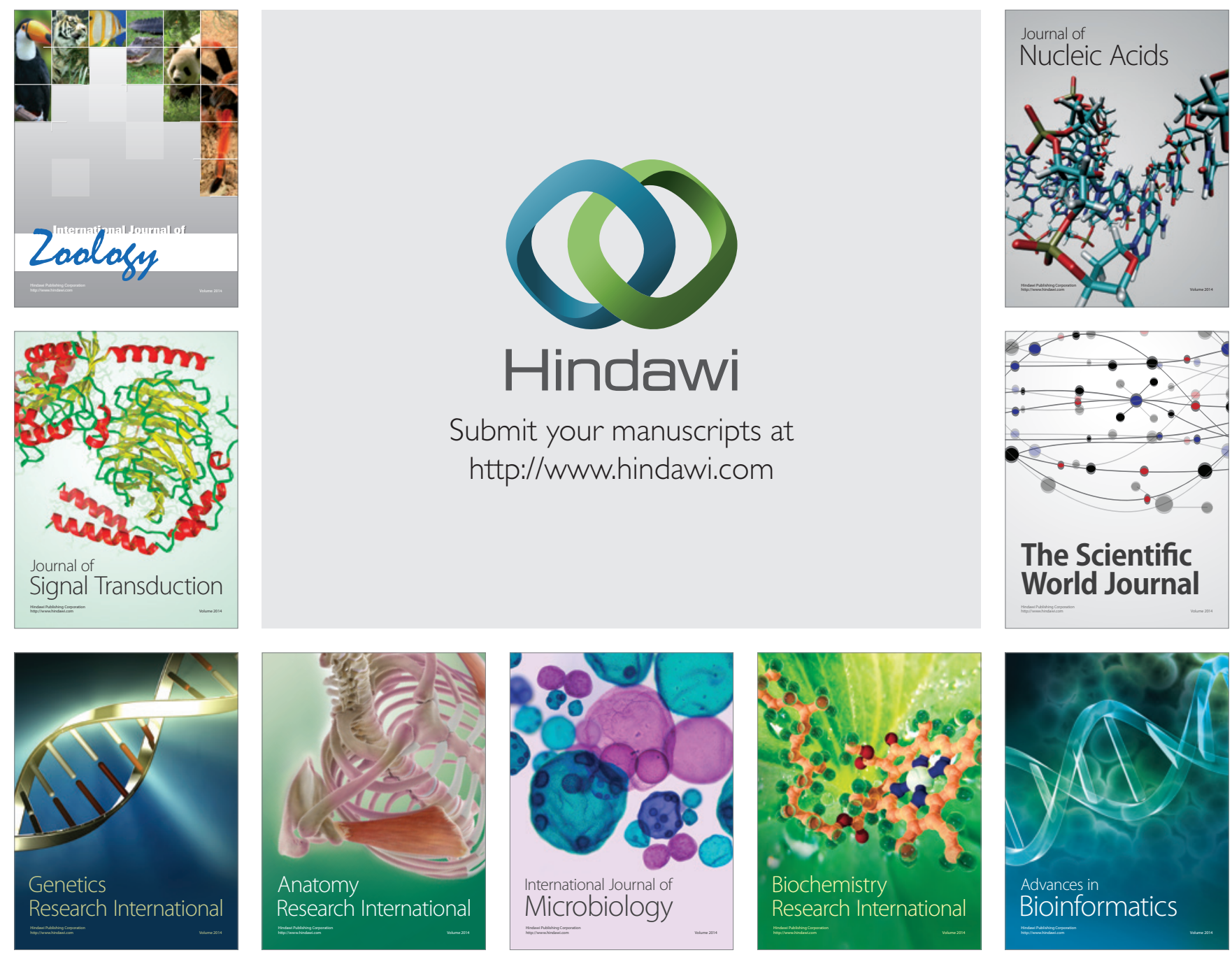

The Scientific World Journal
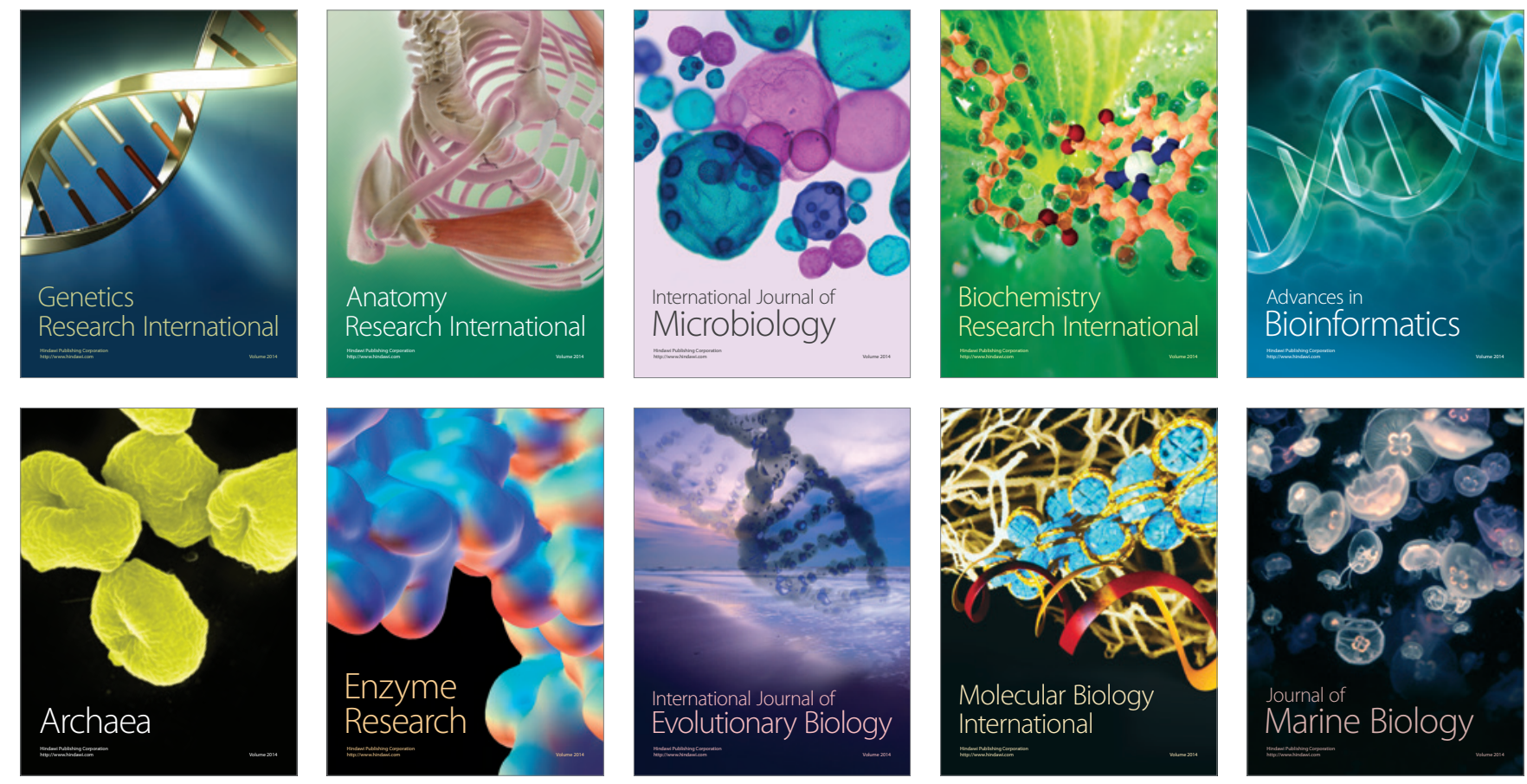